\title{
Modeling and Control of a Suspended Gravity Compensation System with Rigid-Elastic Coupling
}

\author{
Jiao Jia \\ The Seventh Research Division and the Center for Information and Control, School of Automation Science and \\ Electrical Engineering, Beihang University (BUAA) \\ Beijing, 100191, China \\ Yingmin Jia \\ The Seventh Research Division and the Center for Information and Control, School of Automation Science and \\ Electrical Engineering, Beihang University (BUAA) \\ Beijing, 100191, China \\ Shihao Sun \\ The Seventh Research Division and the Center for Information and Control, School of Automation Science and \\ Electrical Engineering, Beihang University (BUAA) \\ Beijing, 100191, China \\ E-mail:annebuaa@hotmail.com;ymjia@buaa.edu.cn;jxcrssh@126.com \\ www.buaa.edu.cn
}

\begin{abstract}
In this paper, a suspension gravity compensation system (SGCS) is modeled and the corresponding controller is designed. The system is a servo platform consisting of three sub-systems by which a micro-gravity environment can be established. Especially, a novel zero stiffness suspension sub-system and a creative unconstrained structure are proposed. The system model is deduced based on Lagrange equation. A PID controller is designed by using the feedback linearization approach. Simulation results show the effectiveness of the proposed method.
\end{abstract}

Keywords: gravity compensation, rigid-elastic coupling, Lagrange method, feedback linearization, PID

\section{Introduction}

One of the vital differences between the space environment and the ground laboratory environment is that the space is micro-gravity. In order to represent the spacecraft motion in space on the ground and improve the fidelity of ground verification of guidance and control system, the micro-gravity environment should be built. Up to now, the gravity compensation methods have been developed such as weight loss, neutral buoyancy, air-bearing and suspension system ${ }^{1-5}$.There are both advantages and disadvantages of these approaches. Among them the suspension method m $^{5-7}$ becomes an economical, practical and reliability method.

There are two kinds of suspension compensation methods passive suspension compensation ${ }^{5}$ and active suspension compensation ${ }^{6-7}$. The active suspension method performs much better than the passive one. The compensation accuracy of the suspend system depends on the structure and controller of the vertical direction sub-system greatly. Usually, a wire rope is one of the 
necessary parts of the vertical direction sub-system. However, when a force which is opposite to or greater than the object gravity functions on the object, the rope is invalid. To solve this problem, a spring is combined with the rope, but the compensation accuracy is still affected by the rope reversing. In this paper, we develop a new means that combines a spring buffer unit with the transmission unit to avoid the shortage of the wire rope. Besides, there are rarely papers or researches that concern how to guarantee the object rotation freedoms when it is suspended to the compensation platform. In this paper, a creative mechanical structure is presented.

\section{System Design}

The platform is a servo system consisting of a levelservo sub-system (LSS), a zero stiffness suspension subsystem (ZSSS), an unconstrained suspension sub-system (USS) and a circuit control section (CCS). The LSS can follow the object's horizontal movement and maintain the ZSSS vertically. The ZSSS could compensate the gravity of the object suspended to the USS and assure its gravity wouldn't affect its movement. The USS holds the object and such that the object would rotate freely. Note that they work cooperatively to create a microgravy environment.

As described in Fig.1.the object connects to the platform through the USS. The USS is composed of twelve rolling bearings and their fixations, which guarantees the equivalent suspension point coincides with the object centroid. Hence, the object can keep balance at any random attitude. The USS links to the spring buffer. The spring buffer attaches to the ZSSS. The ZSSS includes a universal joint, the $\mathrm{Z}$ direction mechanism and the motor_3. The CCS contains a dualaxis tilt sensor, a tension sensor, drive control cards, an acquisition card and a PC. The dual-axis tilt sensor is fixed to the spring buffer through an aluminum panel. The spring buffer and the tension sensor are connected by the universal joint. Therefore, when the object moves, the spring buffer swings around the universal joint. The dual-axis tilt sensor can measure the swing angle. The tension sensor connects to the $Z$ direction mechanism. The $Z$ direction mechanism transmits by a rack and a pinion driven by the motor_3. The ZSSS installs on the LSS specifically on the $\mathrm{Y}$ direction mechanism. In addition, the LSS comprises the motor_2, the $\mathrm{X}$ direction mechanism and the motor_1. The $\mathrm{Y}$ direction mechanism mounts on the $\mathrm{X}$ direction mechanism and they are driven by the motor_2 and the motor_1 respectively. The $\mathrm{X}$ direction mechanism fixes on the supporting frame.

The USS consists of twelve bearings and their fixations as presented in Fig.2. The bearings are classified into yaw bearings, roll bearings and pitch bearings according to their corresponding support rotation of the object.

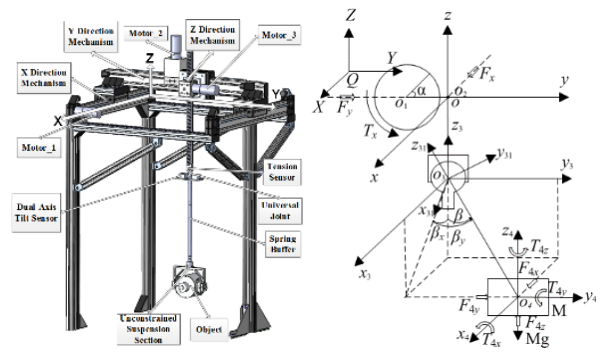

Fig.1.Structure and coordinate system of SGCS

The working principle of the platform is as follows. When the object moves along the horizontal plane the dual-axis tilt sensor can measure the swing angles. Then the motor_1 and motor_2 can drive the $\mathrm{X}$ and $\mathrm{Y}$ direction mechanism to eliminate the swing angle to maintain the spring buffer vertically. When the object moves vertically the length of the spring will vary. The tension sensor will detect the variation and then the motor_3 actuates the $\mathrm{Z}$ direction mechanism to eliminate the variation. So the vertical movement of the object wouldn't be affected by its own gravity. The object is in a micro-gravity environment.

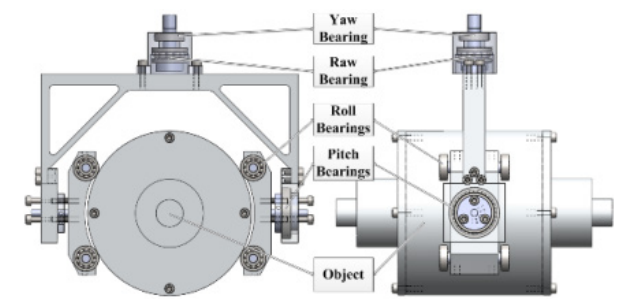

Fig.2. Structure of USS

\section{Model Building}

The SGCS coordinate system is shown in Fig.1.right. Among it, the spring buffer and the application of USS are equivalent to a spring and a rope linked to the centroid of the object respectively. The meaning of each part is as below. 
- $m_{0}$, the load of LSS,

- $\quad m_{1}, m_{2}$, the mass of the pinion and rack resp.,

- $\quad M$, the mass of object,

- $\alpha$, the rotation angle of the pinion

- $Q-X Y Z$, static coordinate system

- $O$, centroid of $m_{0}$

- $O_{1}, O_{2}, O_{4}$, centroids of $m_{1}, m_{2}, M$ resp.,

- $\mathrm{O}_{3}$, centroid of the universal joint,

- $F_{x}, F_{y}$, motor_1\&2 equivalent driving force on $\mathrm{X} \& \mathrm{Y}$

- $T_{x}$, motor_3 output torque on the pinion

- $F_{4 x}, F_{4 y}, F_{4 z}$, the object driving force

- $T_{4 x}, T_{4 y}, T_{4 z}$, the object driving torque

- $\quad \beta$,wing angle between spring buffer $\&$ vertical

- $\beta_{x}, \beta_{y}$, orthogonal decomposition of $\beta$

- $O-x y z$, coordinate moves with $O$,

- $O_{i}-x_{i} y_{i} z_{i}, i=3,4$, coordinates move with $O_{3} \& O_{4}$

- $O_{3}-x_{31} y_{31} z_{31}$, coordinate rotate along the buffer.

From the definition above, the coordinates of $O, O_{1}, O_{2}$ are $(x, y, 0),(x, y-R, 0)$ and $(x, y, \alpha R)$.

And the velocity of each point is $(\dot{x}, \dot{y}, 0),(\dot{x}, \dot{y}, 0)$ and $(\dot{x}, \dot{y}, \dot{\alpha} R)$.

Define $l_{1}=M g / k, d=l_{0}+l_{1}+l$. Then $O_{4}$ is $\left(x+d \sin \beta_{x} \cos \beta_{y}, y+d \sin \beta_{y}, \alpha R-0.5 h_{0}-d \cos \beta_{x} \cos \beta_{y}\right)$

$h_{0}$ is the length of the rack. $O \& O_{2}$ coincide at the initial position. $l$ is the variation length of the spring. Set $l=0$, when the force of the spring is equal to $M g$.

For $\beta \leq 5^{\circ}, \sin \beta \approx \beta, \cos \beta \approx 1$, then

$$
\left(x+d \beta_{x}, y+d \beta_{y}, \alpha R-0.5 h_{0}-d\right)
$$

The velocity of $O_{4}$ is

$$
\left(\dot{x}+\dot{l} \beta_{x}+d \dot{\beta}_{x}, \dot{y}+\dot{l} \beta_{y}+d \dot{\beta}_{y}, \dot{\alpha} R-\dot{l}\right)
$$

The attitudes of the object will be changed as long as one of the $T_{4 x}, T_{4 y}, T_{4 z}$ is valid, and therefore the angular velocity is time-varying. But when we choose $x, y, \alpha, l, \beta_{x} \& \beta_{y}$ as the system generalized coordinates, they are independent of the object rotational kinetic energy. Hence, we could ignore it when we calculate the system kinetic energy.

We define $m_{0}$ as the load of LSS. The pinion and rack move along the $\mathrm{X} \& \mathrm{Y}$ direction mechanism at the horizontal direction, so this part of kinetic energy is included in $0.5 m_{0 x} v_{m 0 x}^{2}+0.5 m_{m 0 y} v_{y}^{2}\left(m_{0 x}, m_{0 y}\right.$ the load of $\mathrm{X}, \mathrm{Y}$ direction resp.).

The system kinetic energy is

$$
\begin{aligned}
T & =\frac{1}{2}\left(\frac{1}{2} m_{1} R^{2}\right) \dot{\alpha}^{2}+\frac{1}{2} m_{2}(\dot{\alpha} R)^{2}+\frac{1}{2} m_{0 x} v_{m 0 x}^{2} \\
& +\frac{1}{2} m_{m 0 y} v_{y}^{2}+\frac{1}{2} M\left(v_{M x}^{2}+v_{M y}^{2}+v_{M z}^{2}\right)
\end{aligned}
$$

Choose $x O y$ plane as zero potential energy surface and the system potential energy is

$$
\begin{gathered}
V=m_{2} g \alpha R+0.5 k\left(l+l_{1}\right)^{2}+M g\left(\alpha R-0.5 h_{0}-d\right) \\
L=T-V
\end{gathered}
$$

Lagrange equation

$$
\frac{d}{d t}\left(\frac{\partial L}{\partial \dot{q}}\right)-\frac{\partial L}{\partial q}=Q_{j}(j=1,2, \ldots \ldots)
$$

Substitute Eq. (1) into Eq. (2) and define

$$
\begin{gathered}
m=\frac{1}{2} m_{1}+m_{2}+M, \eta_{x}=\frac{M+m_{0 x}}{M}, \eta_{y}=\frac{M+m_{0 y}}{M} \\
\eta=\frac{m}{M}, \eta_{2}=\frac{m_{2}+M}{M}, \varsigma=\frac{k}{M}, \rho=\frac{1}{M} \\
\beta^{2}=\beta_{x}^{2}+\beta_{y}^{2}, \beta_{x}^{y}=\beta_{x} \dot{\beta}_{x}+\beta_{y} \dot{\beta}_{y}
\end{gathered}
$$

The equations deduced from Eq. (2) can be formatted in a compact form

$$
\begin{aligned}
& {\left[\begin{array}{cccccc}
\eta_{x} & 0 & 0 & \beta_{x} & d & 0 \\
0 & \eta_{y} & 0 & \beta_{y} & 0 & d \\
0 & 0 & \eta R^{2} & -R & 0 & 0 \\
\beta_{x} & \beta_{y} & -R & \beta^{2}+1 & d \beta_{x} & d \beta_{y} \\
d & 0 & 0 & d \beta_{x} & d^{2} & 0 \\
0 & d & 0 & d \beta_{y} & 0 & d^{2}
\end{array}\right]\left[\begin{array}{c}
\ddot{x} \\
\ddot{y} \\
\ddot{\alpha} \\
\ddot{l} \\
\ddot{\beta}_{x} \\
\ddot{\beta}_{y}
\end{array}\right]+} \\
& {\left[\begin{array}{cccccc}
0 & 0 & 0 & 2 \dot{\beta}_{x} & 0 & 0 \\
0 & 0 & 0 & 2 \dot{\beta}_{y} & 0 & 0 \\
0 & 0 & 0 & 0 & 0 & 0 \\
0 & 0 & 0 & 2 \beta_{x}^{y} & 0 & 0 \\
0 & 0 & 0 & 2 d \dot{\beta}_{x} & 0 & 0 \\
0 & 0 & 0 & 2 d \dot{\beta}_{y} & 0 & 0
\end{array}\right]\left[\begin{array}{c}
\dot{x} \\
\dot{y} \\
\dot{\alpha} \\
i \\
\dot{\beta}_{x} \\
\dot{\beta}_{y}
\end{array}\right]+\left[\begin{array}{c}
0 \\
0 \\
\eta_{2} \mathrm{~g} R \\
\zeta l \\
0 \\
0
\end{array}\right]=\rho\left[\begin{array}{c}
F_{x} \\
F_{y} \\
T_{x} \\
F_{4 z} \\
F_{4 x} \\
F_{4 y}
\end{array}\right]}
\end{aligned}
$$

We classify the system generalized coordinates into two parts based on their controllability that $x, y, \alpha$ only controlled by the motors and the other ones are affected by the driving forces of the object simultaneously. 


$$
\begin{gathered}
\text { Define } q_{1}=\left[\begin{array}{lll}
x & y & \alpha
\end{array}\right]^{T}, q_{2}=\left[\begin{array}{lll}
l & \beta_{x} & \beta_{y}
\end{array}\right]^{T}, \text { and } \\
M_{1} \ddot{q}_{1}+M_{2} \ddot{q}_{2}+C_{1} \dot{q}_{1}+C_{2} \dot{q}_{2}+G_{1}=F_{1} \\
M_{2}^{T} \ddot{q}_{1}+M_{3} \ddot{q}_{2}+C_{3} \dot{q}_{1}+C_{4} \dot{q}_{2}+G_{2}=F_{2}
\end{gathered}
$$

Obviously, $M_{2}$ is invertible

Let $E q .(4)-M_{1} M_{2}^{-T} E q .(5)$. And $C_{1}=C_{3}=0$ we gain

$$
\begin{gathered}
M_{m} \ddot{q}_{2}+C \dot{q}_{2}+G=F_{1}-M_{1} M_{2}^{-T} F_{2} \\
M_{m}=M_{2}-M_{1} M_{2}^{-T} M_{3}, C=C_{2}-M_{1} M_{2}^{-T} C_{4}, \\
G=G_{1}-M_{1} M_{2}^{-T} G_{2}
\end{gathered}
$$

\section{Controller Design}

Since $F_{2}=\left[\begin{array}{lll}F_{4 z} & F_{4 z} & F_{4 z}\end{array}\right]^{T}$ depends on the object movement requirements and is uncontrollable for SGCS, it's regarded as interferences. So the model is a nonlinear time-varying coupling model with disturbances $^{8-10}$. A PID controller is designed by using the feedback linearization approach.

$$
\begin{gathered}
F_{1}=F_{11}+M_{m} F_{12} \\
F_{11}=C \dot{q}_{2}+G \\
F_{12}=-\left(K_{P} q_{2}+K_{I} \int q_{2}+K_{D} \dot{q}_{2}\right) \\
K_{P}=\left[\begin{array}{lll}
K_{P z} & K_{P x} & K_{P y}
\end{array}\right], K_{I}=\left[\begin{array}{lll}
K_{I z} & K_{I x} & K_{I y}
\end{array}\right] \\
K_{D}=\left[\begin{array}{lll}
K_{D z} & K_{D x} & K_{D y}
\end{array}\right]
\end{gathered}
$$

$F_{11}$ is used to counterweigh $C \dot{q}_{2}+G$ of Eq. (6). Substitute Eq. (7) in Eq. (6), and we gain

$$
M_{m} \ddot{q}_{2}=M_{m} F_{12}-M_{1} M_{2}^{-T} F_{2}
$$

It's easy to verify the existence of the inverse of $M_{m}$. Eq. (10) simplifies to

$$
\ddot{q}_{2}=F_{12}+d(\mathrm{t}), d(\mathrm{t})=-M_{1} M_{2}^{-T} F_{2}
$$

Where $F_{12}$ is a PID controller. PID parameters adjustment rules are employed to find suitable control parameters for the system.

\section{Simulation Results}

Firstly, the control target is $q_{2}=0$. It means the gravity of the object is compensated completely.
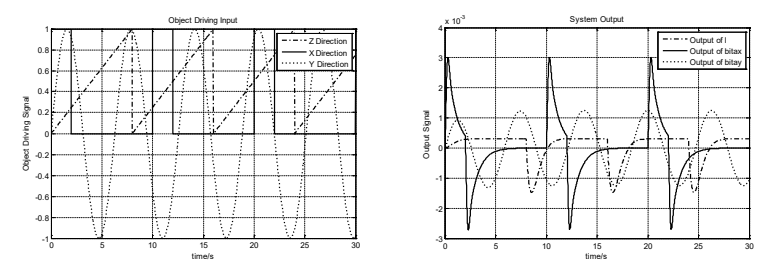

Fig.3. The object driving input and output of the system

The object driving input is shown in Fig.3.left which is regarded as the system disturbance. From Fig.3.right, the system spring variation error is less than $0.0015 \mathrm{~m}$ which means the compensation accuracy is up to $99 \%$.
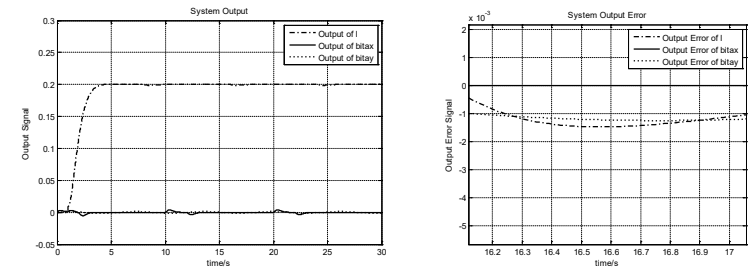

Fig.4. The system response \& output error of the 0.2 step input

Then set $q_{2}=\left[\begin{array}{lll}u(\mathrm{t}) & 0 & 0\end{array}\right]^{T} \cdot u(\mathrm{t})$ is the reference input of the system which means when the spacecraft is not complete weightlessness and it varies according to the law role of the gravity effect.

Fig.4. shows the system response and output error at 0.2 step reference input with the same disturbance as shown in Fig.3.left. The compensation accuracy is up to 99\%. The simulation results show that smaller of spring stiffness coefficient the higher compensation accuracy.

\section{Conclusions}

In this paper, a novel compensation platform reflecting in the ZSSS and USS is proposed and then the mathematic model based on Lagrange equation is developed. Lastly, a stable and high compensation accuracy controller is given. However, the existence of the sensor errors and modeling errors, we can't compensate the nonlinear parts completely. The depth study should be conducted to find more practical controllers $^{11-15}$.

\section{Acknowledgements}

This work was supported by the National Basic Research Program of China (973 Program: 2012CB821200, 2012CB821201) and the NSFC (61134005, 61327807, 61521091, 61520106010). 


\section{References}

1. Y. Watanabe, et al., Microgravity experiments for a visual feedback control of a space robot capturing a target, Proceedings of the IEEE/RSJ International Conference on Intelligent Robots and Systems, (USA, Piscataway, 1998), pp. 1993-1998.

2. H. Sawada, et al., Micro-gravity experiment of a space robotic arm using parabolic flight, Advanced Robotics. 18(3) (2004) 247-267.

3. C. Menon, et al., Issues and solutions for testing freeflying robots, Acta Astronautica. 60(12) (2007) 957-965.

4. A. Robertson, et al., Spacecraft formation flying control design for the Orion mission, AIAA Guidance, Navigation, and Control Conference, (USA, Reston, 1999), pp. 1562-1575.

5. D. A. Kienholz, et al., Very low frequency suspension systems for dynamic testing, Proceedings of the 30th Structures, Structural Dynamics and Materials, (USA, Mobile, 1989), pp. 327-336.

6. G. C. White and Y. S. Xu, Active vertical-direction gravity compensation system, IEEE Transactions on Instrumentation and Measurement. 43(6) (1994) 786792.

7. O. Han, et al., Gravity-offloading system for largedisplacement ground testing of spacecraft mechanisms, Proceedings of 40th aerospace mechanisms symposium, (USA, FL, 2010), pp. 119-132.

8. Y. Jia, Robust Control with Decoupling Performance for Steering and Traction of 4WS Vehicles unde $r$ Velocity-
Varying Motion, IEEE Transactions on Control Systems Technology. 8(3)(2000)554-569.

9. Y. Jia, Alternative Proofs for Improved LMI Representations for the Analysis and the Design of Continuous-Time Systems with Polytopic Type Uncertainty: A Predictive Approach, IEEE Transactions on Automatic Control. 48( 8)(2003) 1413-1416.

10. Y. Jia, General Solution to Diagonal Model Matching Control of Multi - Output-Delay Systems and Its Applications in Adaptive Scheme, Progress in Natural Science. 19( 1)( 2009) 79-90.

11. J. He, and Y. Jia, Adaptive Sliding Mode Control for Magnetic Levitation Vehicles, Journal of Robotics Networking \& Artificial Life. 1(2)(2014)169-173.

12. X. Lu, and Y. Jia, Attitude Reorientation of Spacecraft with Attitude Forbidden Zones, Journal of Robotics Networking \& Artificial Life. 2(1)(2015)13-16.

13. C. Yang, and Y. Jia, Adaptive Multiple-Model Control of A Class of Nonlinear Systems, Journal of Robotics Networking \& Artificial Life. 2(2)(2015)69-72.

14. M. Duan, and Y. Jia, Adaptive Sliding Mode Control for A 2 DOF Magnetic Levitation System with Uncertain Parameters, Journal of Robotics Networking \& Artificial Life. 2(4) (2016)263-267.

15. L. Chen, and Y. Jia, Variable-poled Tracking Control of a Two-wheeled Mobile Robot Using Differential Flatness, Journal of Robotics Networking \& Artificial Life. 1(1)(2014) 12-16. 